\title{
MRI of the temporo-mandibular joint: which sequence is best suited to assess the cortical bone of the mandibular condyle? A cadaveric study using micro-CT as the standard of reference
}

\author{
Karlo, Christoph A ; Patcas, Raphael ; Kau, Thomas ; Watzal, Helmut ; Signorelli, Luca ; Müller, \\ Lukas ; Ullrich, Oliver ; Luder, Hans-Ulrich ; Kellenberger, Christian J
}

\begin{abstract}
OBJECTIVE: To determine the best suited sagittal MRI sequence out of a standard temporomandibular joint (TMJ) imaging protocol for the assessment of the cortical bone of the mandibular condyles of cadaveric specimens using micro-CT as the standard of reference. METHODS: Sixteen TMJs in 8 human cadaveric heads (mean age, 81 years) were examined by MRI. Upon all sagittal sequences, two observers measured the cortical bone thickness (CBT) of the anterior, superior and posterior portions of the mandibular condyles (i.e. objective analysis), and assessed for the presence of cortical bone thinning, erosions or surface irregularities as well as subcortical bone cysts and anterior osteophytes (i.e. subjective analysis). Micro-CT of the condyles was performed to serve as the standard of reference for statistical analysis. RESULTS: Inter-observer agreements for objective $(\mathrm{r}=0.83-0.99, \mathrm{P}<0.01)$ and subjective ( $=0.67-0.88)$ analyses were very good. Mean CBT measurements were most accurate, and cortical bone thinning, erosions, surface irregularities and subcortical bone cysts were best depicted on the 3D fast spoiled gradient echo recalled sequence (3D FSPGR). CONCLUSION: The most reliable MRI sequence to assess the cortical bone of the mandibular condyles on sagittal imaging planes is the 3D FSPGR sequence. KEY POINTS: MRI may be used to assess the cortical bone of the TMJ. • Depiction of cortical bone is best on 3D FSPGR sequences. - MRI can assess treatment response in patients with TMJ abnormalities.
\end{abstract}

DOI: https://doi.org/10.1007/s00330-012-2391-8

Posted at the Zurich Open Repository and Archive, University of Zurich

ZORA URL: https://doi.org/10.5167/uzh-71544

Journal Article

Published Version

Originally published at:

Karlo, Christoph A; Patcas, Raphael; Kau, Thomas; Watzal, Helmut; Signorelli, Luca; Müller, Lukas; Ullrich, Oliver; Luder, Hans-Ulrich; Kellenberger, Christian J (2012). MRI of the temporo-mandibular joint: which sequence is best suited to assess the cortical bone of the mandibular condyle? A cadaveric study using micro-CT as the standard of reference. European Radiology, 22(7):1579-1585.

DOI: https://doi.org/10.1007/s00330-012-2391-8 


\title{
MRI of the temporo-mandibular joint: which sequence is best suited to assess the cortical bone of the mandibular condyle? A cadaveric study using micro-CT as the standard of reference
}

\author{
Christoph A. Karlo • Raphael Patcas • Thomas Kau • \\ Helmut Watzal • Luca Signorelli • Lukas Müller • \\ Oliver Ullrich • Hans-Ulrich Luder • \\ Christian J. Kellenberger
}

Received: 20 October 2011 /Revised: 5 December 2011 / Accepted: 21 December 2011 /Published online: 10 February 2012

(C) European Society of Radiology 2012

\begin{abstract}
Objective To determine the best suited sagittal MRI sequence out of a standard temporo-mandibular joint (TMJ) imaging protocol for the assessment of the cortical bone of the mandibular condyles of cadaveric specimens using micro-CT as the standard of reference.

Methods Sixteen TMJs in 8 human cadaveric heads (mean age, 81 years) were examined by MRI. Upon all sagittal sequences, two observers measured the cortical bone thickness (CBT) of the anterior, superior and posterior portions of
\end{abstract}

C. A. Karlo $(\bowtie)$

Department of Diagnostic and Interventional Radiology,

University Hospital Zurich,

Rämistrasse 100,

8091, Zurich, Switzerland

e-mail: christoph.karlo@gmail.com

C. A. Karlo $\cdot$ T. Kau $\cdot$ H. Watzal $\cdot$ C. J. Kellenberger

Department of Diagnostic Imaging,

University Children's Hospital Zurich,

Zurich, Switzerland

R. Patcas $\cdot$ L. Signorelli $\cdot$ L. Müller

Clinic for Orthodontics and Pediatric Dentistry, Center of Dental

Medicine, University of Zurich,

Zurich, Switzerland

O. Ullrich

Institute of Anatomy, Faculty of Medicine, University of Zurich,

Zurich, Switzerland

H.-U. Luder

Section of Orofacial Structures and Development, Center of Dental Medicine, University of Zurich,

Zurich, Switzerland the mandibular condyles (i.e. objective analysis), and assessed for the presence of cortical bone thinning, erosions or surface irregularities as well as subcortical bone cysts and anterior osteophytes (i.e. subjective analysis). Micro-CT of the condyles was performed to serve as the standard of reference for statistical analysis.

Results Inter-observer agreements for objective $(\mathrm{r}=0.83$ $0.99, P<0.01)$ and subjective $(\kappa=0.67-0.88)$ analyses were very good. Mean CBT measurements were most accurate, and cortical bone thinning, erosions, surface irregularities and subcortical bone cysts were best depicted on the 3D fast spoiled gradient echo recalled sequence (3D FSPGR).

Conclusion The most reliable MRI sequence to assess the cortical bone of the mandibular condyles on sagittal imaging planes is the 3D FSPGR sequence.

Key Points

- MRI may be used to assess the cortical bone of the TMJ.

- Depiction of cortical bone is best on 3D FSPGR sequences.

- MRI can assess treatment response in patients with TMJ abnormalities.

Keywords Mandibular condyle $\cdot$ MRI · Micro-CT · TMJ . JIA

\section{Introduction}

Magnetic resonance imaging (MRI) of the temporomandibular joint (TMJ) is considered the diagnostic imaging technique of choice for the initial workup and follow-up of patients with TMJ abnormalities. These include internal derangements (i.e. deformation or displacement of the 
articular disc), inflammatory conditions (i.e. TMJ arthritis) and degenerative changes (i.e. TMJ arthrosis) $[1,2]$.

The main strengths of MRI are the detailed illustration of soft tissue abnormalities as well as the reliable depiction of bone marrow oedema, undoubtedly an important biomarker for disease progression and treatment response especially in patients suffering from TMJ arthritis [3-8]. As opposed to bone marrow oedema representing an indicator for early bone involvement, flattening of the condylar head, osseous erosions, subchondral bone cysts and anterior osteophytes represent frequent findings in patients with advanced TMJ disease [2].

Although MRI has the ability to illustrate these findings, their true (i.e. in vivo) extent may be uncertain [9]. Based on the close observation of the cortical bone structures of paediatric TMJs in more than 100 MRI examinations performed at our institution per year, the hypothesis arose that the cortical bone structure of the mandibular condyle may present itself differently when different sagittal MRI sequences are used. The reasons for these different appearances are probably chemical shift and susceptibility artefacts occurring at the bordering regions between cortical bone and the adjacent soft tissue. These artefacts usually manifest as a loss of signal at bone-tissue interfaces owing to a dephasing of signals. They usually produce an expanding low signal just beyond the periphery of cortical bone [10-12].

Therefore the purpose of this study was to determine the most suitable sagittal MRI sequence out of our standard TMJ protocol for the evaluation of the cortical bone of the mandibular condyles.

\section{Materials and methods}

\section{Subjects}

Eight intact human cadaveric heads ( 5 female, 3 male; mean age, 81 years; age range, $65-95$ years) were supplied by the Anatomical Institute of the local university in accordance with state and federal regulations (voluntary body donation programme on the basis of informed consent), the Convention on Human Rights and Medicine [13] and the recommendation of the National Academy of Medical Science [14]. Perfusion was carried out within 4 days of decease with a fixation solution consisting of two parts alcohol (70\%), one part glycerine and $2 \%$ almudor (i.e. containing: $8.1 \%$ formaldehyde, $10 \%$ glyoxal and $3.7 \%$ glutaraldehyde).

Image data acquisition

All MRI examinations of all heads $(n=8)$ were carried out on a commercially available 1.5-T MRI unit (Signa HDx,
General Electric, Milwaukee, WI, USA) using a commercially available, two-channel phased array surface coil dedicated to TMJ imaging (DUALTMJ coil). All examinations were performed in closed mouth position. All sagittal sequences were planned to be acquired parallel to the mandibular rami, separately for each side (i.e. left and right). The MRI protocol included the following sagittal sequences: a T1-weighted 2D fast spoiled gradient recalled echo sequence (T1-2D-FSPGR), an intermediate-weighted proton density fast spin echo sequence (PD-FSE), a T2-weighted fast spin echo sequence (T2-FSE), a T1-weighted 3D fast spoiled gradient recalled echo sequence (T1-3D-FSPGR) and a T1-weighted fast spin echo sequence (T1-FSE). For the complete MRI protocol, please refer to Table 1. The mean total examination time was $40 \mathrm{~min}$ per head.

In preparation for the micro-CT $(\mu \mathrm{CT})$ examinations, a member of the Anatomical Institute resected and cleaned all mandibles. Subsequently the mandibular condyles and articular discs were separated from the rami at the level of the mandibular notch (i.e. at the incisura mandibulae). Care was taken not to injure the mandibular condyle during resection.

All $\mu \mathrm{CT}$ examinations of the condyles $(n=16)$ were performed using a commercially available $\mu \mathrm{CT}$ unit (Specimen microCT $\mu$ CT 40, Scanco Medical, Brüttisellen, Switzerland) with all imaging parameters kept identical during all examinations (tube voltage, $70 \mathrm{kV}$, tube current $114 \mu \mathrm{A}$; isotropic resolution, $18 \mu \mathrm{m}$ ).

One radiologist not involved in further data analysis prepared all $\mu \mathrm{CT}$ data by reconstructing multi-planar reformatted (MPR) images in sagittal imaging planes aligned parallel to the mandibular ramus (i.e. corresponding to the alignment of the imaging planes of the sagittal MRI sequences) at a reconstruction slice thickness of $1 \mathrm{~mm}$ and a reconstruction increment of $0.6 \mathrm{~mm}$. Subsequently all reconstructed DICOM data were archived into the hospital's PACS (picture archive and communication system) for storage and further image analysis.

\section{Cortical bone thickness measurements}

The thickness of the cortical bone (CBT) of the anterior, superior and posterior portions of the mandibular condyles was measured on all sagittal MRI sequences at the level of the centre of the mandibular condyle in a blinded fashion by two radiologists experienced in musculoskeletal radiology. All measurements were carried out using a calibrated measurement tool that was part of the hospital's PACS and allowed for sub-millimetre measurements. A third radiologist who was not involved in MRI measurements performed the corresponding CBT measurements on the sagittal MPR images of the $\mu \mathrm{CT}$ data, which served as the standard of reference for statistical analysis. 
Table 1 MRI protocol used for imaging of the temporo-mandibular joints

\begin{tabular}{|c|c|c|c|c|c|c|c|c|}
\hline & $\begin{array}{l}\text { Axial } \\
\text { T2-FRFSE }\end{array}$ & $\begin{array}{l}\text { Coronal } \\
\text { T2-FRFSE }\end{array}$ & $\begin{array}{l}\text { Sagittal } \\
\text { T1-2D-FSPGR }\end{array}$ & $\begin{array}{l}\text { Sagittal } \\
\text { PD-FSE }\end{array}$ & $\begin{array}{l}\text { Sagittal } \\
\text { T2-FSE }\end{array}$ & $\begin{array}{l}\text { Sagittal } \\
\text { T1-3D-FSPGR }\end{array}$ & $\begin{array}{l}\text { Sagittal } \\
\text { T1-FSE }\end{array}$ & $\begin{array}{l}\text { Coronal } \\
\text { T1-SE }\end{array}$ \\
\hline Time to repetition (TR, ms) & 3,000 & 3,000 & 370 & 3,200 & 6,820 & 11.6 & 640 & 500 \\
\hline Time to echo (TE, ms) & 102 & 102 & 4.2 & 24 & 85 & 4.1 & 10.7 & 11 \\
\hline Flip angle $\left(^{\circ}\right)$ & 90 & 90 & 80 & 90 & 90 & 20 & 90 & 90 \\
\hline Matrix (frequency $\times$ phase, pixels) & $384 \times 320$ & $384 \times 320$ & $384 \times 224$ & $256 \times 224$ & $256 \times 224$ & $256 \times 192$ & $256 \times 192$ & $256 \times 192$ \\
\hline Slice thickness (mm) & 3 & 3 & 2 & 2 & 2 & 2 & 2 & 2 \\
\hline Spacing $(\mathrm{mm})$ & 11.6 & 4.1 & 2 & 2 & 2 & 1 & 2 & 2 \\
\hline Field of view (FOV, $\left.\mathrm{cm}^{2}\right)$ & 22 & 22 & 12 & 12 & 12 & 10 & 12 & 16 \\
\hline Band width (Hertz) & 41.67 & 41.67 & 31.25 & 17.86 & 20.83 & 15.63 & 20.83 & 19.23 \\
\hline NEX (number of excitations) & 4 & 4 & 3 & 3 & 4 & 3 & 3 & 3 \\
\hline Echo train length & 21 & 21 & - & 8 & 16 & - & 3 & - \\
\hline
\end{tabular}

FRFSE fast relaxation fast spin echo, FSPGR fast spoiled gradient recalled echo, FSE fast spin echo, SE spin echo

Subjective evaluation of the cortical bone

In a separate analysis session, the same radiologists also assessed the anterior, superior and posterior portions of the mandibular condyles $(n=16)$ on the sagittal MRI sequences for cortical bone thinning, cortical bone erosions, irregularities of the cortical bone surface, subcortical bone cysts and the presence of an anterior osteophyte in a blinded fashion. The third radiologist not involved in MRI analysis assessed the $\mu \mathrm{CT}$ data sets for the same findings to define the standard of reference for statistical analysis. See Fig. 1 for micro-CT imaging examples.

\section{Statistical analysis}

All quantitative variables are described as mean \pm standard deviation. The data were descriptively reviewed and statistically analysed using Kolmogorov-Smirnov's test for normality. $P$ values $<0.05$ were considered statistically significant. All statistical analyses were performed using commercially available software (SPSS, release 17.0, Chicago, IL, USA).
Interobserver agreements regarding the presence of cortical bone thinning, cortical bone erosions, irregularities of the cortical bone surface, subcortical bone cysts and the presence of an anterior osteophyte were analysed using Cohen's kappa $(\kappa)$ statistics and interpreted as follows: A $\kappa$-value greater than 0.81 corresponded to excellent agreement, $0.61-0.80$ to very good interobserver agreement, $0.41-0.60$ to good interobserver agreement and $0.21-0.40$ to moderate interobserver agreement. Interobserver agreements concerning all continuous variables (i.e. measurements of cortical bone thickness) were calculated using interclass correlation coefficients (ICC).

Diagnostic accuracy, sensitivity, specificity, positive predictive value and negative predictive value regarding the assessment of cortical bone thinning, cortical bone erosions, cortical bone surface irregularities and subcortical bone cysts were assessed separately by both observers from chisquared tests of contingency, and the $95 \%$ confidence intervals were calculated. The Wilcoxon signed ranks test was performed to test for statistically significant differences in cortical bone thickness measurements between the sagittal MRI sequence and the $\mu \mathrm{CT}$-based measurements, which served as the standard of reference.
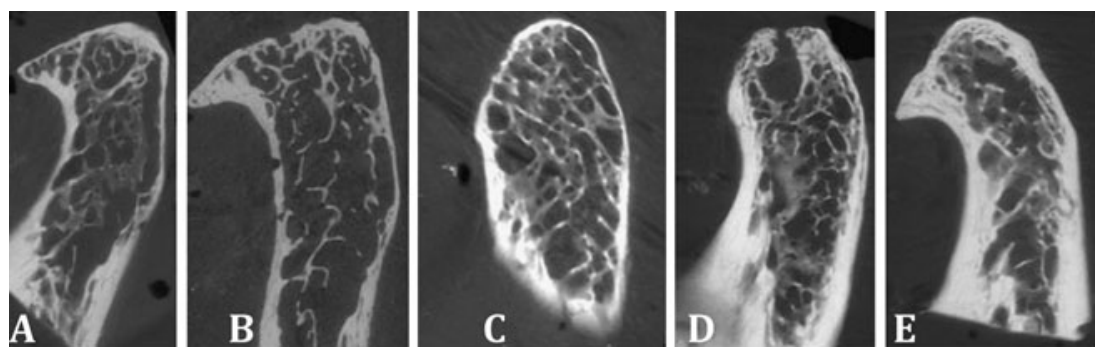

Fig. 1 Micro-CT imaging examples illustrating a cortical bone thinning of the superior portion of the mandibular condyle, $\mathbf{b}$ cortical erosion of the superior portion, $\mathbf{c}$ cortical surface irregularities of the anterior portion, $\mathbf{d}$ a large subcortical bone cyst and $\mathbf{e}$ an anterior osteophyte 


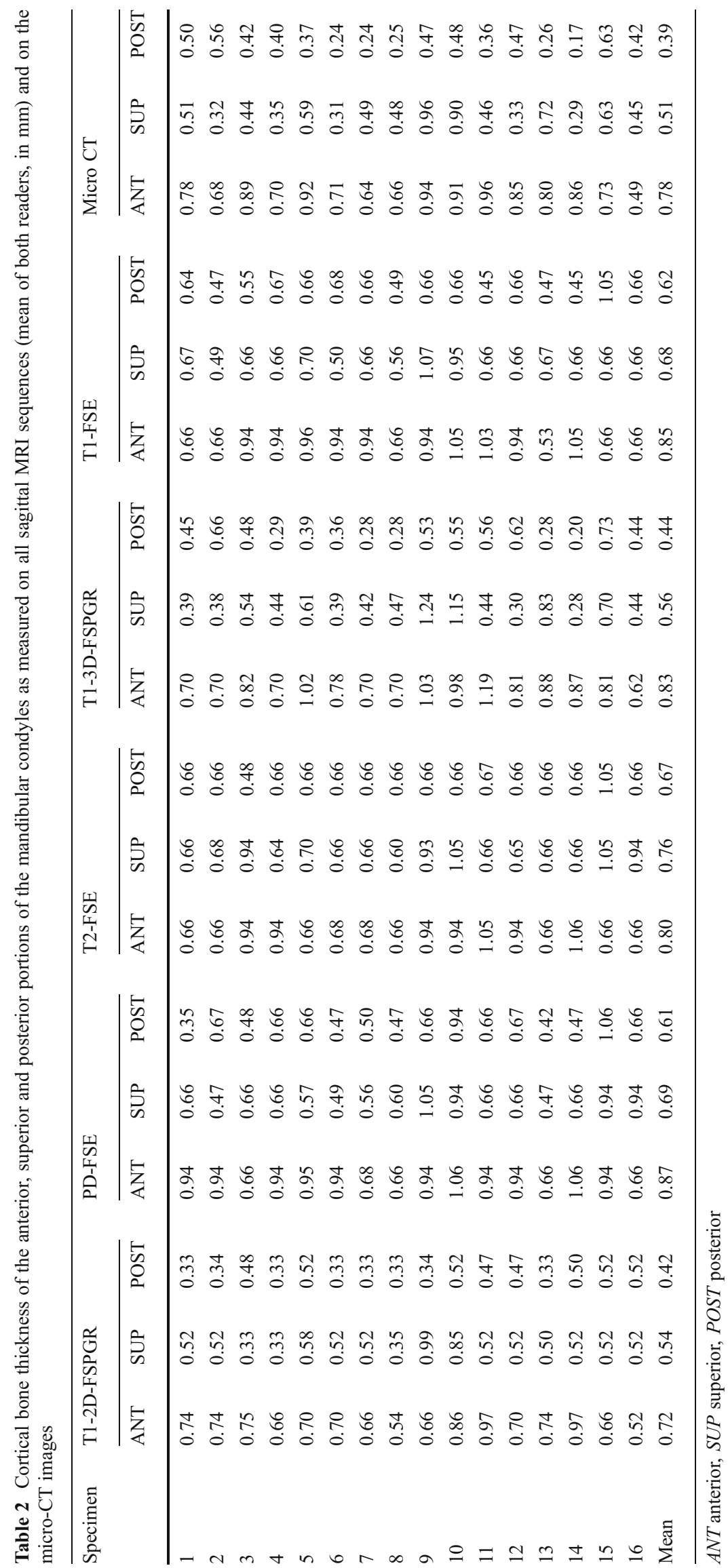




\section{Results}

Imaging findings

The cortical bone of a total of 16 mandibular condyles divided into anterior, superior and posterior portions was investigated (total number of sites $=48$ ). Imaging findings included cortical thinning $(n=16)$, cortical erosions $(n=6)$, cortical surface irregularities $(n=24)$, subcortical bone cysts $(n=3)$ and an anterior osteophyte $(n=4)$.

\section{Inter-observer agreements}

Inter-observer agreements for performing all cortical bone thickness measurements were excellent $(r=0.83-0.99, P<$ 0.01 ). Thus the mean of both observers' measurements was calculated and used for further statistical analyses.

Inter-observer agreements for the detection of cortical bone thinning, cortical bone erosions, cortical bone surface irregularities and subcortical bone cysts ranged from very good to excellent for all locations (i.e. anterior, superior and posterior; $\kappa=0.67-0.85$ ) and all MRI sequences (i.e. T1-2DFSPGR, T2-FSE, T1-3D-FSPGR, PD-FS and T1-FSE: $\kappa=$ $0.74-0.88)$. Inter-observer agreement for the detection of an anterior osteophyte was excellent for all MRI sequences $(\kappa=1.0)$.

\section{Objective analysis}

All descriptive results for cortical bone thickness measurements are summarised in Table 2. Compared with the $\mu \mathrm{CT}$ based measurements, statistically significant differences were found for all cortical bone thickness measurements performed upon the T2-FSE, the PD-FSE and the T1-2DFSPGR sequences (i.e. anterior, superior and posterior portions) as well as the anterior and posterior cortical bone thickness measurements performed upon the T1-FSE (each $P<0.05)$. No statistically significant differences were found for all T1-3D-FSPGR-based measurements (i.e. anterior $[P=0.14]$, superior $[P=0.60]$ and posterior $[P=0.22])$ and for the superior T1-FSE-based measurements $(P=0.16)$ when compared with the $\mu \mathrm{CT}$-based measurements.

\section{Subjective analysis}

Accuracy, sensitivity, specificity, and positive and negative predictive values for the depiction of cortical thinning, cortical erosions, cortical surface irregularities and subcortical bone cysts are illustrated in Table 3. When compared with the $\mu$ CT-based evaluation, the T1-3D-FSPGR sequence was the most reliable in the assessment of cortical thinning, cortical erosions, cortical surface irregularities and subcortical bone cysts for both readers. The depiction of an anterior osteophyte was perfect upon all sequences for both readers. For imaging examples please refer to Figs. 2 and 3.

\section{Discussion}

It was the purpose of this study to determine the most suitable sagittal MRI sequence for the evaluation of the cortical bone of the mandibular condyles. Our results strongly support the T1-weighted 3D fast spoiled gradient recalled echo sequence (i.e. T1-3D-FSPGR) to be the best suited MRI sequence for this task. This sequence may be added to any MRI protocol of the TMJ increasing the total examination time by approximately $6 \mathrm{~min}$.

We discovered significant differences among the evaluated MRI sequences regarding objective and subjective cortical bone assessments. These differences could be attributed

Table 3 Results from subjective analysis (mean of both readers)

\begin{tabular}{|c|c|c|c|c|c|c|c|c|c|c|}
\hline & \multicolumn{5}{|c|}{ Cortical bone thinning } & \multicolumn{5}{|c|}{ Cortical bone surface irregularities } \\
\hline & T1-2D-FSPGR & T2 FSE & T1-3D-FSPGR & PD FSE & T1 FSE & T1-2D-FSPGR & T2 FSE & T1-3D-FSPGR & PD FSE & T1 FSE \\
\hline Accuracy & 0.73 & 0.69 & 0.79 & 0.65 & 0.73 & 0.51 & 0.54 & 0.88 & 0.58 & 0.56 \\
\hline Sensitivity & 0.28 & 0.21 & 0.78 & 0.50 & 0.50 & 0.17 & 0.08 & 0.83 & 0.25 & 0.38 \\
\hline Specificity & 0.91 & 0.88 & 0.79 & 0.71 & 0.82 & 0.94 & 0.96 & 0.92 & 0.92 & 0.75 \\
\hline PPV & 0.57 & 0.42 & 0.61 & 0.41 & 0.54 & 0.80 & 0.67 & 0.91 & 0.75 & 0.60 \\
\hline \multirow[t]{3}{*}{ NPV } & 0.76 & 0.73 & 0.90 & 0.77 & 0.80 & 0.46 & 0.52 & 0.85 & 0.55 & 0.55 \\
\hline & \multicolumn{5}{|c|}{ Cortical bone erosions } & \multicolumn{5}{|c|}{ Subcortical bone cysts } \\
\hline & T1-2D-FSPGR & T2 FSE & T1-3D-FSPGR & PD FSE & T1 FSE & T1-2D-FSPGR & T2 FSE & T1-3D-FSPGR & PD FSE & T1 FSE \\
\hline Accuracy & 0.90 & 0.92 & 0.88 & 0.69 & 0.79 & 0.94 & 0.94 & 1.00 & 0.94 & 0.94 \\
\hline Sensitivity & 0.33 & 0.33 & 0.83 & 0.50 & 0.33 & 0.33 & 0.33 & 1.00 & 0.33 & 0.33 \\
\hline Specificity & 0.98 & 0.99 & 0.88 & 0.71 & 0.86 & 0.98 & 0.98 & 1.00 & 0.98 & 0.98 \\
\hline PPV & 0.67 & 0.67 & 0.50 & 0.20 & 0.25 & 0.50 & 0.50 & 1.00 & 0.50 & 0.50 \\
\hline NPV & 0.91 & 0.93 & 0.97 & 0.91 & 0.90 & 0.96 & 0.96 & 1.00 & 0.96 & 0.96 \\
\hline
\end{tabular}



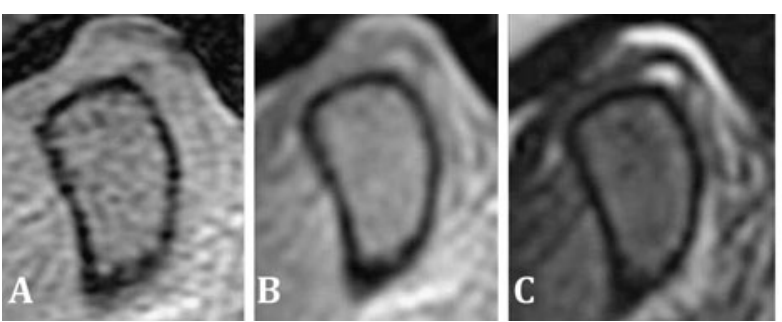
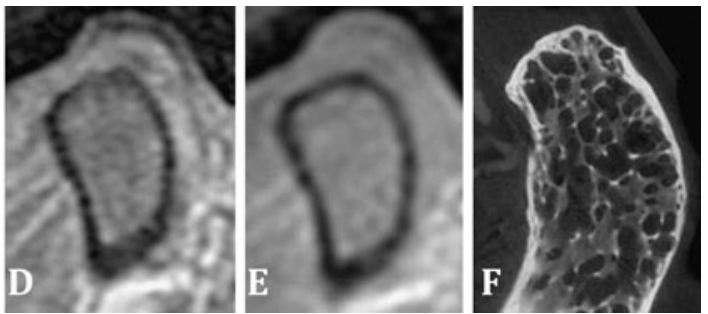

Fig. 2 a 2D fast spoiled gradient recalled echo (2D FSPGR), b intermediate-weighted proton density fast spin echo (PD-FSE), c T2weighted fast spin echo (T2-FSE), d T1-weighted 3D fast spoiled gradient recalled echo (T1-3D-FSPGR) and e T1-weighted fast spin

to previously described and well-known chemical shift and susceptibility artefacts occurring at the bordering regions between cortical bone and cartilage, fluid or soft-tissue structures of the TMJ $[10-12,15-19]$. The resulting overor underestimation, however, may lead to misinterpretation of the cortical bone surface and structure. Therefore, TMJ imaging protocols should be designed carefully to avoid such errors.

Over the last few years, the demand for cortical bone imaging using MRI has increased. As opposed to computed tomography (CT), which has been considered the imaging technique of choice for the depiction of osseous pathological features so far, MRI operates without applying ionising radiation to the patients. Continuous advances in technology (i.e. higher magnetic field strengths, more efficient software) and the ongoing effort of the musculoskeletal research community have elevated MRI to become the new imaging method of choice for the assessment of cortical bone, especially for serial followup studies in young patients. Various study groups have contributed to cortical bone imaging recently [20-22]. Louis et al. have demonstrated the power of highresolution T1-weighted 3.0-T MRI in quantifying the cortical bone cross-sectional area at the level of the tibia in a comparison with quantitative CT [20]. Stehling et al. reported the delineation of the cortical bone of the echo sequences (T1-FSE). The cortical surface irregularities of the superior portion confirmed by $\mathbf{f}$ micro $\mathrm{CT}$ are depicted by the T1-3DFSPGR sequence only and seem to be absent on all other sequences

mandibular condyle to be significantly better on images derived from a 3.0-T MRI system rather than a $1.5-\mathrm{T}$ MRI system [22].

When performing MRI of the TMJ it is essential to evaluate the structure, thickness and shape of the cortical bone of the mandibular condyle. In patients with TMJ arthritis, especially in young patients and children suffering from juvenile idiopathic arthritis (JIA), the assessment of these cortical bone structures becomes even more important because cortical bone thinning, flattening of the mandibular condyle, the development of subchondral cysts and the presence of anterior osteophytes are regarded as biomarkers for MRI monitoring of the activity and possible progression of JIA $[5,7,8]$.

Abramowicz et al. reported various pathological findings of the mandibular condyle such as erosions, articular surface flattening, subchondral sclerosis and osteophytes [4]. In particular the assessment of subchondral sclerosis may be challenged in this content because false-positive results may be acquired due to chemical shift artefacts. However, Abramowicz et al. evaluated the mandibular condyles upon T1weighted and intermediate proton-density-weighted MR sequences. We were able to show significant differences in cortical bone thickness of the mandibular condyle between those two sequences, thus yielding a potential source of error for the assessment of subchondral sclerosis and cortical bone structure.
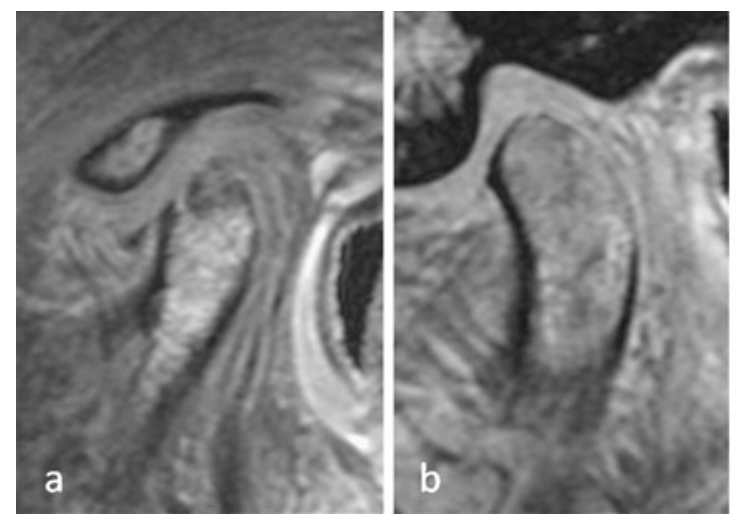

Fig. 3 Imaging example illustrating the ability of the T1-3D-FSPGR sequence to depict a subcortical bone cysts, b osseous erosions, c

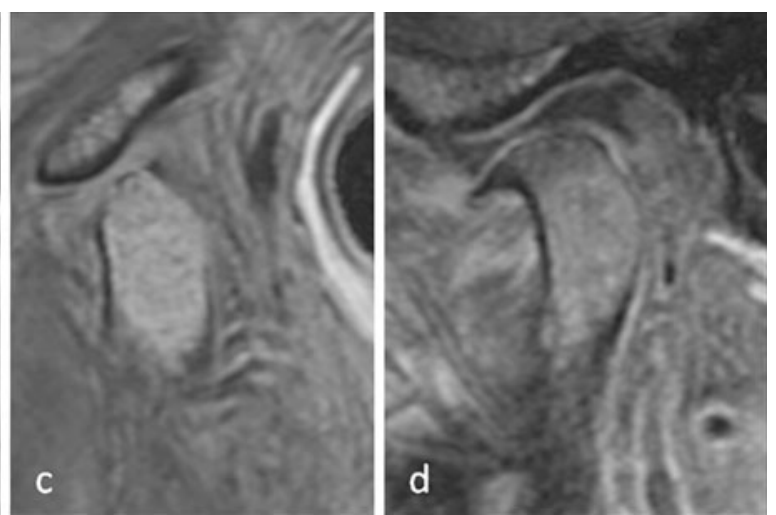

cortical bone surface irregularities and $\mathbf{d}$ the presence of an anterior osteophyte and cortical bone thinning of the superior portion 
In a very recent publication on dynamic contrastenhanced MRI of the TMJ, Tasali et al. described condylar sclerosis as one of their MRI findings [15]. However, no further details were provided on the definition of sclerosis and how it was assessed during image analysis. Because of the variable presentation of cortical bone on different sagittal MRI sequences, false-positive findings may become difficult to avoid.

Our study has some limitations. First, the number of evaluated specimens is low $(n=16)$. However, for the chosen approach relying on cadaveric material this was the maximum number of specimens that could be obtained. Second, we limited our evaluation to the sagittal imaging plane. For the declared objective of our study, we considered the restriction appropriate as the sagittal imaging planes are the most important imaging planes in the MRI assessment of the TMJ.

In conclusion, our study showed that the T1-weighted 3D FSPGR sequence was the most suitable MRI method for the objective and subjective assessments of the cortical bone of the mandibular condyle.

Acknowledgements The authors would like to thank L. Völlmer, M. Studhalter, B. Werner and A. Jezler for their technical and logistical assistance.

\section{References}

1. Magnusson T, Egermark I, Carlsson GE (2000) A longitudinal epidemiologic study of signs and symptoms of temporomandibular disorders from 15 to 35 years of age. J Orofac Pain 14:310-319

2. Moen K, Hellem S, Geitung JT, Skartveit L (2010) A practical approach to interpretation of MRI of the temporomandibular joint. Acta Radiol 51:1021-1027

3. Cannizzaro E, Schroeder S, Muller LM, Kellenberger CJ, Saurenmann RK (2011) Temporomandibular joint involvement in children with juvenile idiopathic arthritis. J Rheumatol 38:510-515

4. Abramowicz S, Cheon JE, Kim S, Bacic J, Lee EY (2011) Magnetic resonance imaging of temporomandibular joints in children with arthritis. J Oral Maxillofac Surg 69:2321-2328

5. Müller L, Kellenberger CJ, Cannizzaro E et al (2009) Early diagnosis of temporomandibular joint involvement in juvenile idiopathic arthritis: a pilot study comparing clinical examination and ultrasound to magnetic resonance imaging. Rheumatology (Oxford) 48:680-685

6. Lewis EL, Dolwick MF, Abramowicz S, Reeder SL (2008) Contemporary imaging of the temporomandibular joint. Dent Clin North Am 52:875-890
7. Boyesen P, Haavardsholm EA, van der Heijde D et al (2011) Prediction of MRI erosive progression: a comparison of modern imaging modalities in early rheumatoid arthritis patients. Ann Rheum Dis 70:176-179

8. Lee EY, Sundel RP, Kim S, Zurakowski D, Kleinman PK (2008) MRI findings of juvenile psoriatic arthritis. Skeletal Radiol 37:987-996

9. McGibbon CA, Bencardino J, Yeh ED, Palmer WE (2003) Accuracy of cartilage and subchondral bone spatial thickness distribution from MRI. J Magn Reson Imaging 17:703-715

10. Reichert IL, Benjamin M, Gatehouse PD et al (2004) Magnetic resonance imaging of periosteum with ultrashort TE pulse sequences. J Magn Reson Imaging 19:99-107

11. Phan CM, Matsuura M, Bauer JS et al (2006) Trabecular bone structure of the calcaneus: comparison of MR imaging at 3.0 and 1.5 T with micro-CT as the standard of reference. Radiology 239:488-496

12. McGibbon CA, Dupuy DE, Palmer WE, Krebs DE (1998) Cartilage and subchondral bone thickness distribution with MR imaging. Acad Radiol 5:20-25

13. European Union (2002) Additional protocol to the convention on human rights and biomedicine, on transplantation of organs and tissues of human origin. ETS 186, Article 16-18

14. Swiss Academy of Medical Science (2008) Verwendung von Leichen und Leichenteilen in der medizinischen Forschung sowie Aus-, Weiter- und Fortbildung. Medical-Ethical Guidelines and Recommendations, pp 1-11

15. Tasali N, Cubuk R, Aricak M et al (2011) Temporomandibular joint (TMJ) pain revisited with dynamic contrast-enhanced magnetic resonance imaging (DCE-MRI). Eur J Radiol. doi:10.1016/j. ejrad.2011.01.044

16. McGibbon CA (2003) Inter-rater and intra-rater reliability of subchondral bone and cartilage thickness measurement from MRI. Magn Reson Imaging 21:707-714

17. McGibbon CA, Bencardino J, Palmer WE (2003) Subchondral bone and cartilage thickness from MRI: effects of chemical-shift artifact. MAGMA 16:1-9

18. Peh WC, Chan JH (2001) Artifacts in musculoskeletal magnetic resonance imaging: identification and correction. Skeletal Radiol 30:179-191

19. Zand KR, Reinhold C, Haider MA, Nakai A, Rohoman L, Maheshwari S (2007) Artifacts and pitfalls in MR imaging of the pelvis. J Magn Reson Imaging 26:480-497

20. Louis O, Cattrysse E, Scafoglieri A, Luypaert R, Clarys JP, de Mey J (2010) Accuracy of peripheral quantitative computed tomography and magnetic resonance imaging in assessing cortical bone cross-sectional area: a cadaver study. J Comput Assist Tomogr 34:469-472

21. Issever AS, Link TM, Newitt D, Munoz T, Majumdar S (2010) Interrelationships between 3-T-MRI-derived cortical and trabecular bone structure parameters and quantitative-computed-tomographyderived bone mineral density. Magn Reson Imaging 28:1299-1305

22. Stehling C, Vieth V, Bachmann R et al (2007) High-resolution magnetic resonance imaging of the temporomandibular joint: image quality at 1.5 and 3.0 Tesla in volunteers. Invest Radiol $42: 428-434$ 